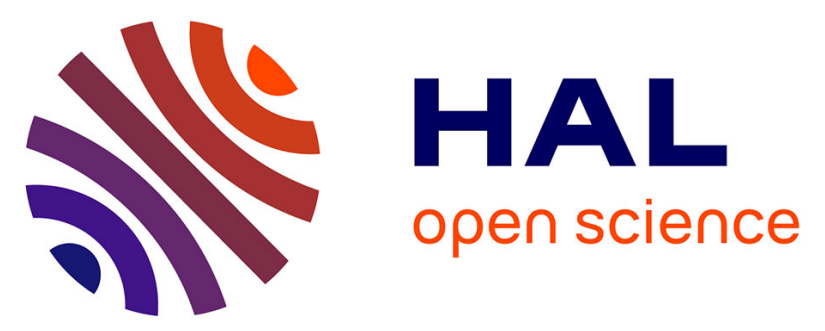

\title{
Determination of the viscous and thermal characteristic lengths of plastic foams by ultrasonic measurements in helium and air
}

Philippe Leclaire, Luc Kelders, Walter Lauriks, Manuel Melon, Niven Brown, Bernard Castagnede

\section{To cite this version:}

Philippe Leclaire, Luc Kelders, Walter Lauriks, Manuel Melon, Niven Brown, et al.. Determination of the viscous and thermal characteristic lengths of plastic foams by ultrasonic measurements in helium and air. Journal of Applied Physics, 1996, 80 (4), pp.2009. 10.1063/1.363817 . hal-01326774

\section{HAL Id: hal-01326774 \\ https://hal.science/hal-01326774}

Submitted on 5 Jun 2016

HAL is a multi-disciplinary open access archive for the deposit and dissemination of scientific research documents, whether they are published or not. The documents may come from teaching and research institutions in France or abroad, or from public or private research centers.
L'archive ouverte pluridisciplinaire HAL, est destinée au dépôt et à la diffusion de documents scientifiques de niveau recherche, publiés ou non, émanant des établissements d'enseignement et de recherche français ou étrangers, des laboratoires publics ou privés. 


\title{
Determination of the viscous and thermal characteristic lengths of plastic foams by ultrasonic measurements in helium and air
}

\author{
Ph. Leclaire, L. Kelders, and W. Lauriks \\ Laboratorium voor Akoestiek en Thermische Fysica, Departement Natuurkunde, \\ Katholieke Universiteit Leuven, Celestijnenlaan 200D, 3001 Leuven, Belgium
}

M. Melon, N. Brown, and B. Castagnède

Laboratoire d'Acoustique du l'Université de Maine, URA CNRS 1101, Avenue O. Messiaen, 72017 Le Mans, France

\begin{abstract}
The acoustic wave most commonly transmitted and detected in the high-porosity absorbent materials used in noise control is generally the air-borne slow compressional wave. In a new experiment, the air saturating the sample is replaced by helium and the transmission is studied at ultrasonic frequencies $(70-600 \mathrm{kHz})$. The experiment is quite easily performed using standard ultrasonics and vacuum equipment. The main purpose of this work is to propose a method to determine simultaneously both the viscous and thermal characteristic lengths with the same precision. These two parameters characterize the viscous and the thermal interactions between the frame and the fluid at high frequencies. The characteristic lengths are deduced from the highfrequency asymptotic behavior of either the velocity or the attenuation curves obtained in the sample saturated by air and by helium. It also appears that due to the properties of helium, the discrepancy previously observed between predictions and measurements is shifted toward higher frequencies.
\end{abstract}

\section{INTRODUCTION}

A very particular prediction of the theory established by Biot $^{1}$ in 1956 on the acoustics of fluid-saturated porous media is the existence of a second compressional wave in consolidated media. At low frequencies, this wave is strongly attenuated and does not propagate, i.e., its velocity tends to zero as the frequency tends to zero. Above a characteristic frequency, which depends on the fluid viscosity and the permeability, the wave becomes propagatory and its attenuation varies as the square root of frequency. The experimental confirmation of this prediction was done by Plona ${ }^{2}$ in an artificial rock made of sintered glass beads saturated by water but no confirmation in normal conditions in real rocks has been performed. Indeed, a slow wave can be generated in natural rocks saturated with superfluid helium He II or He IV because of the very particular properties of these fluids. Such experiments were made to confirm the theory and to determine all the necessary parameters, ${ }^{3-5}$ and to probe porous media. ${ }^{6,7}$ These experiments are not easy to implement for a common laboratory although they look attractive. In most of the cases, the slow wave cannot be easily observed because it is strongly dispersive at low frequencies and highly attenuated at higher frequencies although it is propagative. Nevertheless, Nagy, Adler, and Bonner ${ }^{8}$ have shown that it is possible with a standard experimental setup to detect a slow compressional wave in both artificial and natural rocks saturated by air in a frequency window where the mode is propagative and not too heavily attenuated. This window is rather narrow for air-saturated materials because of the high kinematic viscosity $\nu$ of air. It is set by the condition that the viscous skin depth, $\delta=\sqrt{2 \nu / \omega}$ at the angular frequency $\omega$, must be less than the pore size while the wavelength must be greater than the grain size. ${ }^{8}$

\section{SLOW WAVE PROPAGATION IN HIGH-POROSITY PLASTIC FOAMS}

Recent developments have been carried out in the noise control research field. In many circumstances, a strong attenuation of the slow wave is a great advantage for sound absorption. A rigorous description of the wave propagation in absorbent materials has been proposed, ${ }^{9}$ new materials such as polymeric foams are studied, and techniques have been developed to measure their tortuosity ${ }^{10}$ and characteristic lengths. ${ }^{11-13}$ These materials have generally a high porosity (more than 95\%) and a tortuosity fairly close to one. At audible and ultrasonic frequencies, one of the compressional waves propagates mainly in air in the porous material and the frame remains motionless. The other compressional wave propagates in both media, but its velocity is close to the velocity of compressional waves in the frame in vacuum. This is due to the fact that an acoustic field in air in the porous material cannot generate vibrations of the frame, which is too heavy, but vibrations of the frame can induce a displacement of air. It is more appropriate to call these waves air- and frame-borne waves, but traditionally the air-borne wave, which is highly damped at low frequencies due to the viscous interactions with the frame, is called the slow wave. Indeed, this slow compressional wave is very commonly excited and detected in such materials. The problem of excessive attenuation is not so crucial as for fluid- or gas-saturated rocks because of the high porosity. The slow wave can even be easily detected in many cases and especially in reticulated foams, i.e., with open cells. The permeability of these samples is fairly high and the main part of the incident energy is transmitted as the solid proportion is small. Elsewhere, at high frequencies, an acoustic wave in air outside the material will generate only the air-borne wave in the 
material, leaving the frame motionless and the approximation of a rigid frame can be made. We show that the slow compressional wave can also be detected in high-porosity foams saturated with helium instead of air.

The main contribution of this work is to give a method providing simultaneously the viscous $\Lambda$ and thermal $\Lambda^{\prime}$ characteristic lengths from the exploitation of the dispersion curves in air- and helium-filled materials. These physical parameters have a major importance. They were introduced by Johnson, Koplik, and Dashen ${ }^{14}$ and by Champoux and Allard ${ }^{15}$ for the description of the high-frequency acoustical behavior of fluid-saturated porous media without making any assumption on the pore geometry. Until now, the thermal characteristic length $\Lambda^{\prime}$ which is namely two times the average volume-to-surface ratio of the pores, has been evaluated by a nonacoustic method from the measurement of the specific area using the standard Brunauer-Emmet-Teller (BET) technique. ${ }^{11}$ The viscous characteristic length $\Lambda$ has been recently evaluated from the high-frequency asymptotic behavior of the velocity curve ${ }^{12}$ or of the attenuation curve. ${ }^{13}$ The major disadvantage of these methods is that $\Lambda^{\prime}$ is required and must be determined before. The precision of $\Lambda$ is rather poor as it depends on the precision of $\Lambda^{\prime}$ which is about $20 \%$ with the BET method. Furthermore, $\Lambda$ and $\Lambda^{\prime}$ are not evaluated in the same experimental conditions. In the method proposed here, $\Lambda$ and $\Lambda^{\prime}$ are evaluated simultaneously, with the same precision and on the same area in the sample. The values of $\Lambda$ and $\Lambda^{\prime}$ obtained are therefore relevant only in the area of the sample where they are measured. Problems of inhomogeneity in the porosity, tortuosity, or permeability are not considered here and must be tackled through a systematic study involving a $C$ scan over the surface of the sample.

\section{METHOD PRINCIPLE}

The principle of the method consists of utilizing the difference in physical properties of air and helium in conjunction with the high-frequency asymptotic behavior of the dispersion curves. In the high-frequency limit and in the approximation of a rigid frame, the complex wave number is given by ${ }^{16}$

$$
k=\frac{\omega}{c_{0}} \sqrt{\alpha_{\infty}}\left[1+(1-j) \frac{\delta}{2}\left(\frac{1}{\Lambda}+\frac{\gamma-1}{B \Lambda^{\prime}}\right)\right],
$$

where $\alpha_{\infty}$ is the tortuosity, $c_{0}$ the sound speed in the gas, $B^{2}$ the Prandtl number, and $\gamma$ the specific heat ratio. Two methods are used to extract $\Lambda$ and $\Lambda^{\prime}$ from this expression. The first method ( $\mathrm{Sl}$ ) consists of plotting the real part of the propagation index, i.e., the squared ratio of the reference velocity and the phase velocity in the sample as a function of the square root of the inverse frequency $f^{-1 / 2}$ (Ref. 12). A linear behavior is obtained at high frequencies; the slope leads to a value of the length $L=\left[\Lambda^{-1}\right.$ $\left.+(\gamma-1)\left(B \Lambda^{\prime}\right)^{-1}\right]^{-1}$ while the intercept with the ordinate axis gives the tortuosity $\alpha_{\infty}$. This plot is made for the experimental data obtained with air first and then for those ob- tained with helium. This must lead to two distinct values of the slope and only one value of $\alpha_{\infty}$. The lengths $\Lambda$ and $\Lambda^{\prime}$ are determined solving the system

$$
\begin{aligned}
& \mathrm{Sl}_{\mathrm{air}}=\alpha_{\infty}\left(\sqrt{\nu_{\mathrm{air}} / \pi}\right) / L_{\mathrm{air}}, \\
& \mathrm{Sl}_{\mathrm{He}}=\alpha_{\infty}\left(\sqrt{\nu_{\mathrm{He}} / \pi}\right) / L_{\mathrm{He}},
\end{aligned}
$$

where $\mathrm{Sl}_{\text {air }}$ and $\mathrm{Sl}_{\mathrm{He}}$ are the slopes measured when saturating the sample with air and helium, respectively. The second method $(Q \delta)$, suggested by Johnson and co-workers, ${ }^{14}$ consists of plotting the product of the quality factor and the viscous skin depth $Q \delta$ as a function of frequency providing directly a value of $L$ in the high-frequency limit. ${ }^{13}$ The parameters $\Lambda$ and $\Lambda^{\prime}$ are then deduced from the system,

$$
\lim _{\omega \rightarrow \infty}(Q \delta)_{\text {air }}=L_{\text {air }}, \quad \lim _{\omega \rightarrow \infty}(Q \delta)_{\mathrm{He}}=L_{\mathrm{He}} .
$$

\section{EXPERIMENTAL PROCEDURE}

The practical implementation of the method can easily be done. Experiments are made in air and helium using standard equipment. Pulses of about $350 \mathrm{~V}$ amplitude are provided by a function generator coupled to a power amplifier. The detection system is composed of a charge amplifier connected to a digital oscilloscope. Ultrasonic waves are generated and detected in the two gases by capacitive transducers with 6- $\mu \mathrm{m}$-thick Mylar films as vibrating membranes. The transducers' diameters are $1.5 \mathrm{~cm}$ for the experiments in air and $1 \mathrm{~cm}$ for the experiments in helium in order to favor higher frequencies. The transmission experiments in air are analogous to those of Refs. 12 and 13. The procedure for an experiment in helium is described as follows: The sample and the transducers are enclosed in a vacuum chamber of about $10 \ell$. The distance between the transducers is of a few centimeters and the sample is placed between them in the configuration of normal incidence. A primary vacuum is made to a pressure of more or less than 0.5 Torr so that the proportion of air remaining is less than $0.07 \%$. Then the chamber is filled with helium to the atmospheric pressure and the transmission experiments are made. No problem of saturation is expected since the sample has open cells and a

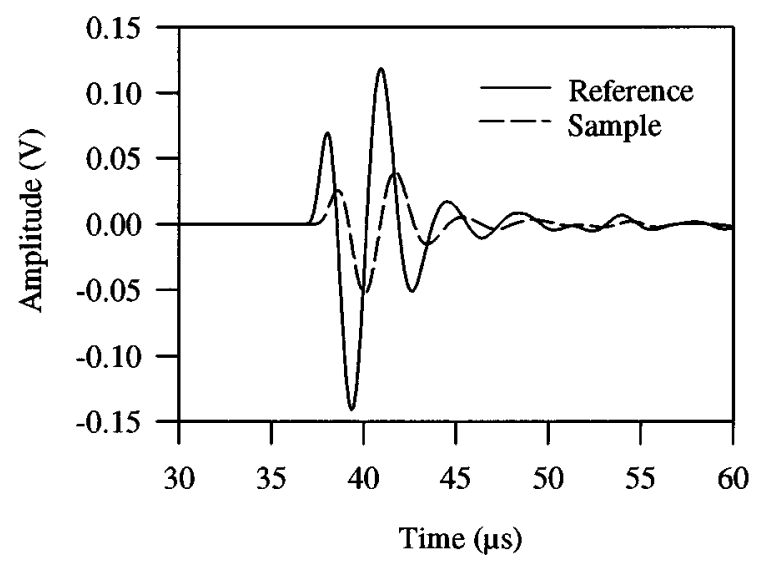

FIG. 1. Reference signal and signal transmitted in a high-porosity foam saturated by helium. 
TABLE I. Sample characteristics.

\begin{tabular}{lccc}
\hline \hline \multicolumn{1}{c}{ Sample } & $\mathrm{s} 1$ & $\mathrm{~s} 2$ & $\mathrm{~s} 3$ \\
\hline Thickness $(\mathrm{mm})$ & 10.0 & 8.8 & 8.8 \\
Porosity & 0.98 & 0.97 & 0.97 \\
Permeability $\left(\mathrm{m}^{2}\right)$ & $3.08 \times 10^{-9}$ & $2.06 \times 10^{-9}$ & $6.47 \times 10^{-9}$ \\
\hline \hline
\end{tabular}

high porosity. For each experiment two signals are captured: the signal crossing the sample and the reference signal obtained after removing the sample. A simple "trick' is used to do the removal: A small piece of metal is fixed on the sample so that it can be easily displaced from outside the vacuum chamber with the help of a simple magnet. The velocity of the slow wave is deduced as a function of frequency from the phase difference between the reference signal and the transmitted signal. The attenuation is obtained from the ratio of the amplitude spectra.

\section{RESULTS}

Figure 1 shows the reference signal and the signal transmitted in the sample s1 when the saturating gas is helium. The quantity $(\gamma-1) / B$ is equal to 0.815 in helium and 0.475 in air. Using these numerical values and assuming that the order of magnitude of $\Lambda^{\prime}$ is approximately double that of $\Lambda$, it can be calculated from Eq. (1) that the ratio $V_{\mathrm{He}} / V_{\text {air }}$ of the limit velocities of the slow wave when the sample is saturated by helium and by air is approximately 2.9 while the ratio of attenuation is about 1.1 [Eq. (1)]. As a result, the slow wave can be detected in the material saturated by helium as well as by air as shown in Fig. 1 but the arrival times are approximately three times less.

Three samples were studied; their useful characteristics are summarized in Table I. The methods $(\mathrm{Sl})$ and $(Q \delta)$ previously described were applied to determine $\alpha_{\infty}, \Lambda$, and $\Lambda^{\prime}$. The numerical values for the sample s1 are presented in this

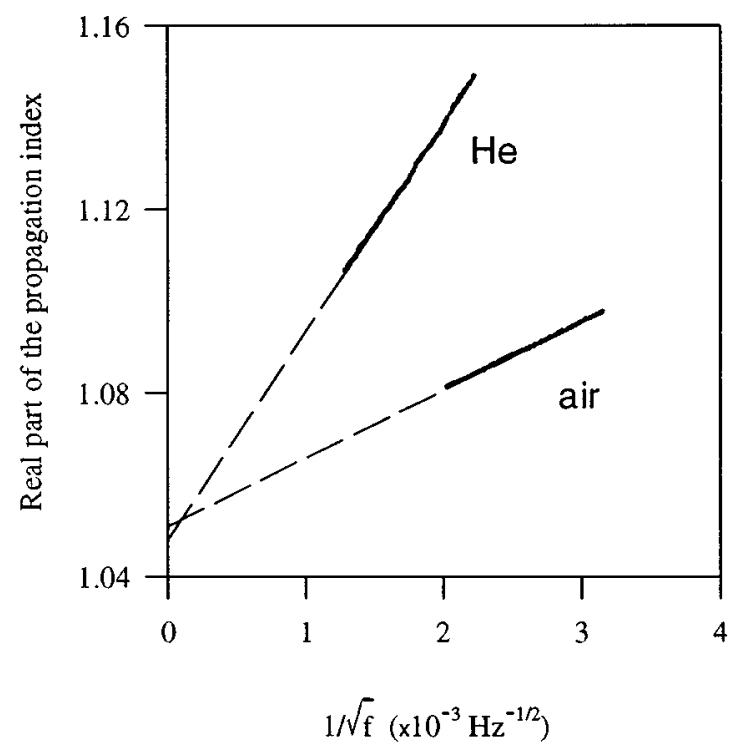

FIG. 2. Real part of the propagation index as a function of the square root of the inverse frequency.

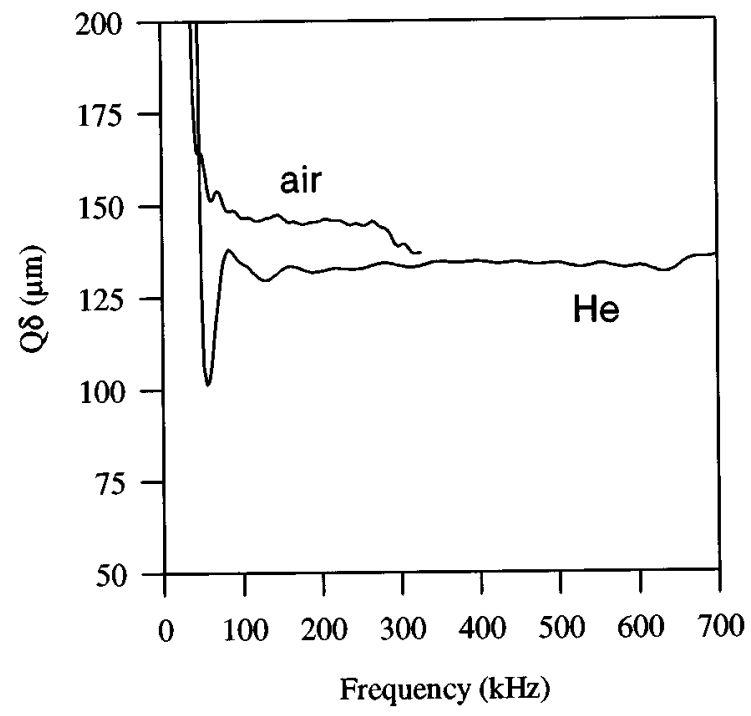

FIG. 3. Product of the quality factor and the viscous skin depth as a function of frequency.

paragraph; the results are discussed in the following paragraph. The real part of the propagation index is plotted in Fig. 2 as a function of $f^{-1 / 2}$ for the sample s1 when it is saturated with helium and with air. A linear behavior is obtained as expected. In this example, the values of $\Lambda$ and $\Lambda^{\prime}$ deduced from the slopes of the curves are, respectively 202 and $367 \mu \mathrm{m}$. A tortuosity of 1.052 is deduced from the intercept while the $Y$ axis in air, and of 1.048 in helium. In order to implement the second method $(Q \delta)$, the product $Q \delta$ is plotted in Fig. 3 as a function of frequency for a sample saturated by air and by helium. The values of $\Lambda$ and $\Lambda^{\prime}$ deduced from the high-frequency limit of $Q \delta$ in air and in helium are, respectively, 180 and $429 \mu \mathrm{m}$.

The results are summarized in Table II. About five experiments were performed for each sample in air and in helium. The values of $\alpha_{\infty}$ were obtained using the Sl method. After averaging, they were found to be about $0.7 \%$ less when working in helium than when working in air. The dispersion being higher in helium, the signals were treated slightly differently than in air. This may explain the small systematic error observed. The parameter $\Lambda^{\prime}$ has also been evaluated using the BET technique. A good agreement is found between the values of $\Lambda^{\prime}$ given by the $S 1$ and $Q \delta$ methods with a maximum discrepancy of $15 \%$ for the sample s1. When comparing the BET method to the acoustic methods ( $\mathrm{Sl}$ and $Q \delta$ ), the maximum difference is $15 \%$ for the sample s $3,24 \%$

TABLE II. Numerical values of $\alpha_{\infty}, \Lambda$, and $\Lambda^{\prime}$ determined by different methods.

\begin{tabular}{lrrr}
\hline \hline \multicolumn{1}{c}{ Sample } & \multicolumn{1}{c}{$\mathrm{s} 1$} & $\mathrm{~s} 2$ & $\mathrm{~s} 3$ \\
\hline$\alpha_{\infty}(\mathrm{Sl})$ & 1.052 & 1.042 & 1.054 \\
$\Lambda^{\prime}(\mathrm{BET})(\mu \mathrm{m})$ & 610 & 370 & 750 \\
$\Lambda^{\prime}(\mathrm{Sl})(\mu \mathrm{m})$ & 367 & 318 & 672 \\
$\Lambda^{\prime}(Q \delta)(\mu \mathrm{m})$ & 429 & 292 & 650 \\
$\Lambda(\mathrm{Sl})(\mu \mathrm{m})$ & 202 & 134 & 273 \\
$\Lambda(Q \delta)(\mu \mathrm{m})$ & 180 & 132 & 249 \\
\hline \hline
\end{tabular}


for the sample s2, and $50 \%$ for the sample s1. These results can be considered to be not too bad as only an order of magnitude is given by the BET method. In addition, the measurements are not made with rigorously the same samples and in the same experimental conditions with the BET method than with the acoustic methods. Comparing the S1 and $Q \delta$ method for the determination of $\Lambda$, the maximum difference is $12 \%$ for the sample s1. The Sl method gives simultaneously a value of $\alpha_{\infty}, \Lambda$, and $\Lambda^{\prime}$, but it is sensitive to the windowing and noise because it requires the determination of phase differences. The $Q \delta$ method is less sensitive to windowing but provides only values of $\Lambda$ and $\Lambda^{\prime}$ and, furthermore, this method breaks down when additional attenuation phenomena occur as seen in Fig. 3 where a "fall down" can be observed from a frequency of about $250 \mathrm{kHz}$ for the curve that corresponds to the experiment in air. Nagy ${ }^{17}$ has proposed two mechanisms that may explain the excess attenuation: the scattering effect, and the losses due to friction on the surface of the material when the viscous skin depth is of the same order as the surface roughness. This mechanism decreases the dynamic permeability of the material. The sound velocity is 2.9 times higher in helium than in air and the kinematic viscosity of helium 8.2 times higher. For a given frequency, the wavelength $\lambda$ and the viscous skin depth $\delta$ are therefore multiplied approximately by a factor of 3 in helium. Consequently, both scattering effects and losses due to surface roughness are expected to appear at higher frequencies. It should be noted that microscopy of reticulated foams does not show any evidence of roughness for the structure. ${ }^{18}$ The excess attenuation observed in air is therefore considered to be mainly due to scattering. The frequency for which the scattering appears will be as lower as the size of the scatterer (the diameter of the fibers for fibrous materials) is greater. As a consequence, the acoustic methods can be used for foams with thin solid fibers.

\section{CONCLUSION}

We have proposed a method to determine both the viscous and thermal characteristic lengths of absorbent materials, saturated by two different gases, from the measurement of the asymptotic behavior of the velocity and attenuation curves in the high frequency limit. The two parameters are measured simultaneously in the same experimental conditions and with the same precision. A good agreement is found between the two acoustic methods ( $\mathrm{Sl}$ and $Q \delta$ ) for the three samples studied. The difference between the BET and acoustic methods is higher; it is explained by the fact that the BET method is rather inaccurate and that samples are not studied in the same experimental conditions. The excess attenuation already observed by Nagy ${ }^{17}$ limits the applicability of the acoustics methods to materials with low diameter solid fibers.

\section{ACKNOWLEDGMENTS}

We are grateful to Professor J. F. Allard for his comments on earlier versions of this article. This work was supported by the SAVANTE Program of the European Union, H. C. M. Contract No. CHRX-CT93-0397.

${ }^{1}$ M. A. Biot, J. Acoust. Soc. Am. I and II 28, 168 (1956).

${ }^{2}$ T. J. Plona, Appl. Phys. Lett. 36, 259 (1980).

${ }^{3}$ D. L. Johnson, T. J. Plona, C. Scala, F. Pasierb, and H. Kojima, Phys. Rev. Lett. 49, 1840 (1982).

${ }^{4}$ D. Singer, F. Pasierb, R. Ruel, and H. Kojima, Phys. Rev. B 30, 2909 (1984).

${ }^{5}$ S. R. Baker and I. Rudnik, in Proceedings of the IEEE Ultrasonics Symposium, 1985, p. 1039.

${ }^{6}$ D. L. Johnson, D. L. Hemmick, and H. Kojima, J. Appl. Phys. 76, 104 (1994).

${ }^{7}$ D. L. Johnson, T. J. Plona, and H. Kojima, J. Appl. Phys. 76, 115 (1994).

${ }^{8}$ P. B. Nagy, L. Adler, and B. P. Bonner, Appl. Phys. Lett. 56, 2504 (1990).

${ }^{9}$ J. F. Allard, Sound Propagation in Porous Media: Modelling Sound Absorbing Materials (Elsevier, New York, 1994).

${ }^{10}$ J. F. Allard, B. Castagnède, M. Henry, and W. Lauriks, Rev. Sci. Instrum. 65, 754 (1994).

${ }^{11}$ M. Henry, P. Lemarinier, J. F. Allard, J. L. Bonardet, and A. J. Gédéon, J. Appl. Phys. 77, 17 (1995).

${ }^{12}$ N. Brown, M. Melon, B. Castagnède, V. Montembault, W. Lauriks, and P. Leclaire, C. R. Acad. Sci. Paris 322, Série IIb, 393 (1996).

${ }^{13}$ P. Leclaire, L. Kelders, W. Lauriks, C. Glorieux, and J. Thoen, J. Acoust. Soc. Am. 99, 1944 (1996).

${ }^{14}$ D. L. Johnson, J. Koplik, and R. Dashen, J. Fluid Mech. 176, 379 (1987).

${ }^{15}$ Y. Champoux and J. F. Allard, J. Appl. Phys. 70, 1975 (1991).

${ }^{16}$ D. Lafarge, J. F. Allard, B. Brouard, C. Verhaegen, and W. Lauriks, J. Acoust. Soc. Am. 93, 2474 (1993).

${ }^{17}$ P. B. Nagy, J. Acoust. Soc. Am. 93, 3224 (1993).

${ }^{18}$ J. F. Allard (personal communication). 